\title{
SARS-CoV 灭活疫苗制备及小鼠免疫研究
}

唐 琳 ${ }^{(1)}$ 汪 建 $^{\left(\mathbb{1}^{*}\right.}$ 秦鄂德 ${ }^{(2)}$ 祝庆余 ${ }^{(2)}$ 于 曼 ${ }^{(2)}$ 石慧颖 ${ }^{(3)}$ 丁志芬 ${ }^{(3)}$ 程晓洁 ${ }^{(1)}$ 王彩平 ${ }^{(1)}$ 李双利 ${ }^{\mathbb{1}}$ 常国辉 ${ }^{\mathbb{1}}$ 张旭敏 ${ }^{(1)}$ 陈希曙 ${ }^{\mathbb{1}}$ 于 军 $^{(1)}$ 陈 则 $^{\left(\mathbb{C}^{(} *\right.}$

(1)中国科学院北京基因组研究所, 北京 101300; (2)军事医学科学院微生物流行病研究所, 北京 100071; 3)卫生部北京生物制品研究 所, 北京 100024; (4)中国科学院武汉病毒研究所, 武汉 430071; (5)湖南师范大学生命科学学院, 长沙 410081.* 联系人, E-mail: chenze@mail.hunnu.edu.cn)

\begin{abstract}
摘要 用 Vero 细胞大量增殖 SARS-CoV 病毒, 使用 $\beta$-丙内酯作为灭活剂灭活病毒后, 用 Sepharose $4 \mathrm{FF}$ 层析柱纯化病毒颗粒得到灭活疫苗, 并经 Western-blot, 质谱, ELISA 和电子显微镜检测证实. 用纯化的 灭活疫苗加入或不加入氢氧化铝佐剂, 以不同的剂量免疫小鼠, 能够诱导小鼠产生抗体, 加入氢氧化铝 佐剂组所产生的抗体高于不加入佐剂组.
\end{abstract}

\section{关键词 SARS 冠状病毒 灭活疫苗 疫苗制备 佐剂}

严重急性呼吸系统综合征(Severe Acute Respiratory Syndrome, SARS)是近几十年来所遇到的最严 重的病毒性疾病之一, 它是由一种新型冠状病毒引起 的急性呼吸道传染病, 具有人群普遍易感、症状严 重、病死率高达 6\% 7\%的特点. 目前研究显示其传 播途径主要是呼吸道飞沫以及黏膜接触感染 ${ }^{[1 \sim 5]}$. 依 据人类控制传染病的经验，疫苗接种应该是预防 SARS 流行的最有效措施 ${ }^{[6 \sim 9]}$. 我们首先选择灭活疫 苗作为我们研制有效抗 SARS 疫苗的第一步. 本文介 绍了用 Vero 细胞培养 SARS 冠状病毒, 以及纯化、 制备灭活疫苗的工艺. 并用制备的灭活疫苗免疫动 物观察其诱导产生抗体的能力.

\section{1 材料和方法}

(i ) 毒种和细胞. 军事医学科学院微生物流行 病研究所提供 SARS-CoV BJ01 株 ${ }^{[10]}$, 此毒株从北京 SARS 患者中分离, 经全基因组测序鉴定为 SARS 病 原体, 其序列已提交 GenBank; Vero 细胞由北京生物 制品研究所提供, 为疫苗生产专用细胞株, 符合 WHO 规定的疫苗生产细胞要求和我国 2000 版《中国 生物制品规程》的要求.

(ii) 疫苗制备. SARS-CoV BJ01病毒株接种于 Vero 细胞, $37^{\circ} \mathrm{C}$ 无血清培养基培养 $36 \mathrm{~h}, 5 \mathrm{~mL} /$ 瓶, 达 $10^{7} \mathrm{TCID}_{50}$ 时, 整瓶细胞 $-20{ }^{\circ} \mathrm{C}$ 冻融 1 次, 6000 $\mathrm{r} / \mathrm{min}$ (Beckman, 25R)离心 $20 \mathrm{~min}$, 取上清作病毒灭活. 在病毒悬液中加人 $1: 2000$ 稀释的 $\beta$-丙内脂(购自 Sigma 公司), 摇匀后置 $4^{\circ} \mathrm{C}$ 孵育 $24 \sim 72 \mathrm{~h}$, 然后置于 $37^{\circ} \mathrm{C} 2 \mathrm{~h}$ 以水解 $\beta$-丙内脂. 灭活后的病毒悬液于 $4^{\circ} \mathrm{C}$ $6000 \mathrm{r} / \mathrm{min}$ (Beckman, 25R)离心 $30 \mathrm{~min}$, S取上清, 以
PEG20000(购自 Sigma 公司)浓缩至 $1 / 20$ 体积, 用 Centriplus YM-100(购自 Millipore公司)进行离心浓缩 $(30000 \times g, 20 \mathrm{~min})$, 过 Sepharose 4FF 层析柱 $(2.6 \mathrm{~cm} \times$ $95 \mathrm{~cm}$ )进行凝胶过滤 $(\mathrm{PBS} 0.8 \mathrm{~mL} / \mathrm{min}$ ) 并对各组分进 行 SDS-PAGE 电泳分析. 将病毒颗粒收集管合并, 继 续行 Centriplus-20 滤管离心浓缩病毒颗粒. 上柱前后 的样品以 Lowry 法测定总蛋白含量, 然后立即加人 $1 \%$ 人白蛋白作稳定剂, 并加人 $0.01 \%$ 硫柳录防腐. 取 适量纯化后样品进行电子显微镜、Western blot、ELISA 和质谱检定. 用 PBS 调整免疫的抗原剂量; 含氢氧化 铝佐剂纯抗原的制备是在 $4{ }^{\circ} \mathrm{C}$ 条件下完成, 氢氧化铝 佐剂(北京生物制品研究所提供, 终浓度 $0.6 \mathrm{mg} / \mathrm{mL}$ )加 至抗原溶液, 振荡 $1 \mathrm{~h}$ 使抗原和佐剂均匀吸附.

(iii) 实验动物和动物免疫实验. 6 周龄䧳性 $\mathrm{BAL} \mathrm{B} / \mathrm{c}$ 小鼠购自北京维通利华实验动物技术有限 公司, 然后将小鼠分组, 各组按不同的剂量免疫灭活 疫苗、灭活疫苗加氢氧化铝佐剂. 用腹腔注射的方式 免疫小鼠, 共免疫 2 次, 间隔 3 周, 第 1 次免疫后 3 周，加强免疫前，用眼眶取血法取血，第 2 次免疫后 1 周, 用 $1 \mathrm{~mL}$ 一次性注射器从心脏取血, 收集血清检 测 $\operatorname{IgG}$ 抗体. 其抗体效价用 ELISA 方法测定, 反应在 聚苯乙烯酶标板上进行, 按顺序依次加人包被液、封 闭液(PBS 中含 $1 \%$ 的 BSA 和 $0.01 \%$ 的叠氮钠)、按 2 倍稀释的血清、生物素标记的山羊抗鼠 $\mathrm{IgG}$ 抗体 $(\gamma$-chain specific)(Southern Biotechnology Associates, Inc. USA)、250 倍稀释的链菌蛋白碱性磷酸酶、0.01 $\mathrm{g} / \mathrm{mL}$ 的 PNPP 溶液, 最后通过酶标仪 (Labsystems Muliskan Ascent, Thermo Bioanalysis Company, Finland)读取波长 $4142405 \mathrm{~nm}$ 下的读数. 


\section{2 结果}

\subsection{SARS-CoV 灭活疫苗制备}

将 SARS-CoV BJ01 株接种 Vero 细胞, 收集病毒 液后进行灭活、浓缩和凝胶过滤处理. 从图 1 可看出, 凝胶过滤时出现两个峰, 第 1 峰电子显微镜检查(图 2)为 $100 \mathrm{~nm}$ 左右, 表面有典型冠状突起的病毒颗粒, 表明为 SARS-CoV 颗粒峰, 第 2 峰为大分子杂蛋白, 电子显微镜下未见 SARS-CoV 颗粒. 最后将病毒颗粒 收集管合并, 继续进行离心浓缩, 获得 SARS-CoV 灭 活疫苗.

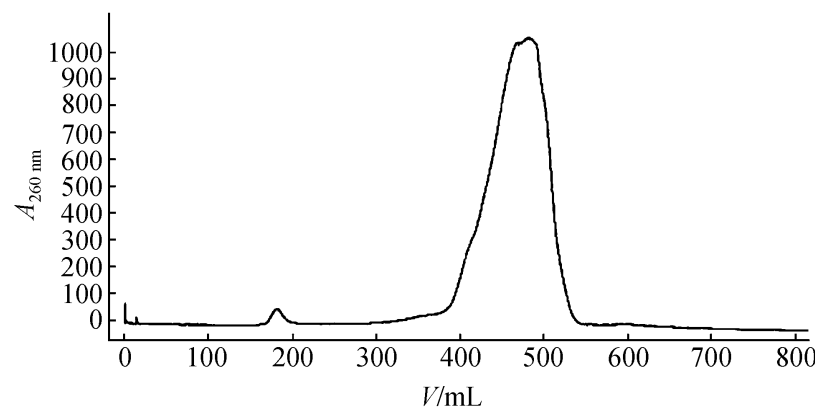

图 1 Sepharose 4FF 分子篮层析柱纯化病毒的层析图谱 在 PBS 缓冲体系中, 浓缩后的灭活病毒上清液经 Sepharose 4FF 分子

篮层析柱进行分离. 用 AKTA purifier 层析工作站控制流速在 0.8 $\mathrm{mL} / \mathrm{min}$, 监测 $260 \mathrm{~nm}$ 下光吸收值的变化. 以 $5 \mathrm{~mL} /$ 管为一个组分进行 收集

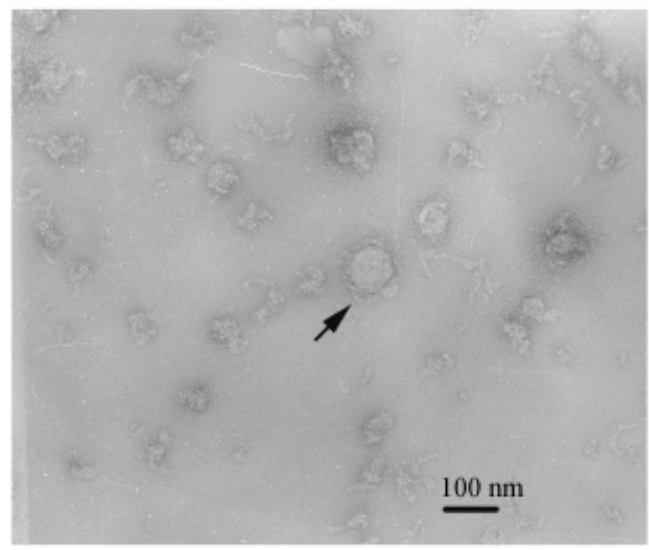

图 2 电子显微镜下的灭活疫苗(病毒颗粒)

\section{2 灭活疫苗残余活病毒检测}

采用 $\beta$-丙内脂 $1: 2000$ 和 $1: 4000,4{ }^{\circ} \mathrm{C}$ 灭活 SARS-CoV; 分别在 $20,24,28,42,48,66,72$ h 取样, 并于 $37^{\circ} \mathrm{C}$ 水解 $\beta$-丙内脂 $2 \mathrm{~h}$, 取 $3 \mathrm{~mL}$ 进行 Vero 细胞臭
传 3 代实验，测定其残毒活性；结果显示出 $1 ： 2000$, $24 \mathrm{~h}$ 能达到灭活效果; 1 : 4000, $28 \mathrm{~h}$ 可达到灭活效果.

\subsection{SDS-PAGE 和 Western Blot 鉴定}

纯化后的病毒颗粒进行 SDS-PAGE(图 3(a))并转 膜, 以北京地区 SARS 患者阳性血清为一抗, AP 标记 的羊抗人 $\operatorname{IgG}$ 为二抗进行 Western blot 检测(图 3(b)). 对照蛋白分子量标记可见, 阳性血清反应条带主要 集中在 50 和 $140 \mathrm{kD}$ 左右, 分别为 $\mathrm{N}$ 蛋白和 $\mathrm{S}$ 蛋白.

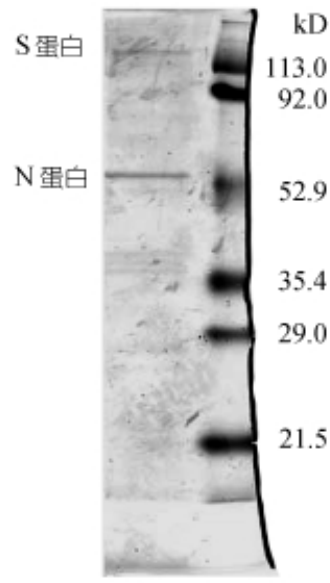

(a)

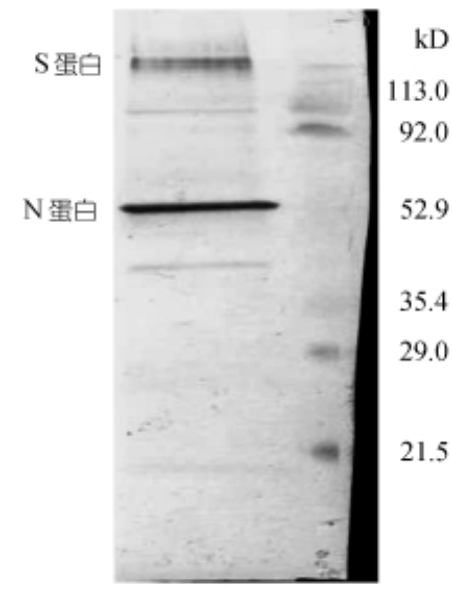

(b)
图 3 聚丙烯酰胺凝胶电泳(SDS-PAGE)(a)和免疫印迹法 (Western blot)(b)显示灭活疫苗中 $S$ 和 $N$ 蛋白 纯化后的病毒在 $12 \%$ SDS-PAGE(a)上电泳分离后, 电转移到 PVDF(聚 偏二氟乙烯)膜上, 以含 5\%脱脂奶粉的 TTBS 溶液(Tris $100 \mathrm{mmol} / \mathrm{L}$, $\mathrm{NaCl} 120 \mathrm{mmol} / \mathrm{L}, \mathrm{pH} 7.9,0.1 \%$ Tween-20) $37^{\circ} \mathrm{C}$ 封闭 $30 \mathrm{~min}$; SARS 患 者血清用 TTBS 溶液 $1: 1000$ 稀释作为一抗 $4{ }^{\circ} \mathrm{C}$ 䁔育过夜, 用 TTBS 溶 液漂洗 3 次后, 加人碱性磷酸酶标记的羊抗人 $\operatorname{IgG}$ (TTBS, 1:1000 稀 释), 用 $\mathrm{NBT} / \mathrm{BCIP}$ 进行显色(b)

\section{4 质谱鉴定}

截取上述 SDS-PAGE 所示 $\mathrm{N}$ 蛋白和 S 蛋白条带 进行 MALDI-TOF 检测, 质谱数据经 NCBI 冠状病毒 数据库检索确定为 $\mathrm{BJ} 01$ 株的 $\mathrm{N}$ 蛋白和 $\mathrm{S}$ 蛋白(图 4 和 5). 质谱显示 $\mathrm{N}$ 蛋白和 $\mathrm{S}$ 蛋白分子量分别为 46 和 $141 \mathrm{kD}$.

\subsection{ELISA 检测}

纯化后的灭活疫苗(病毒颗粒)进行高浓度尿素(7 $\mathrm{mol} / \mathrm{L}$ )超声溶解后包被 ELISA 96 孔板, 用 SARS 冠状 病毒 IgG 抗体检测试剂盒进行检测(北京华大吉比爱 生物技术有限公司提供), 以已知的北京地区 SARS 患者阳性血清作抗体, 证实呈强阳性反应. 正常人对 1 照血清呈阴性结果。 

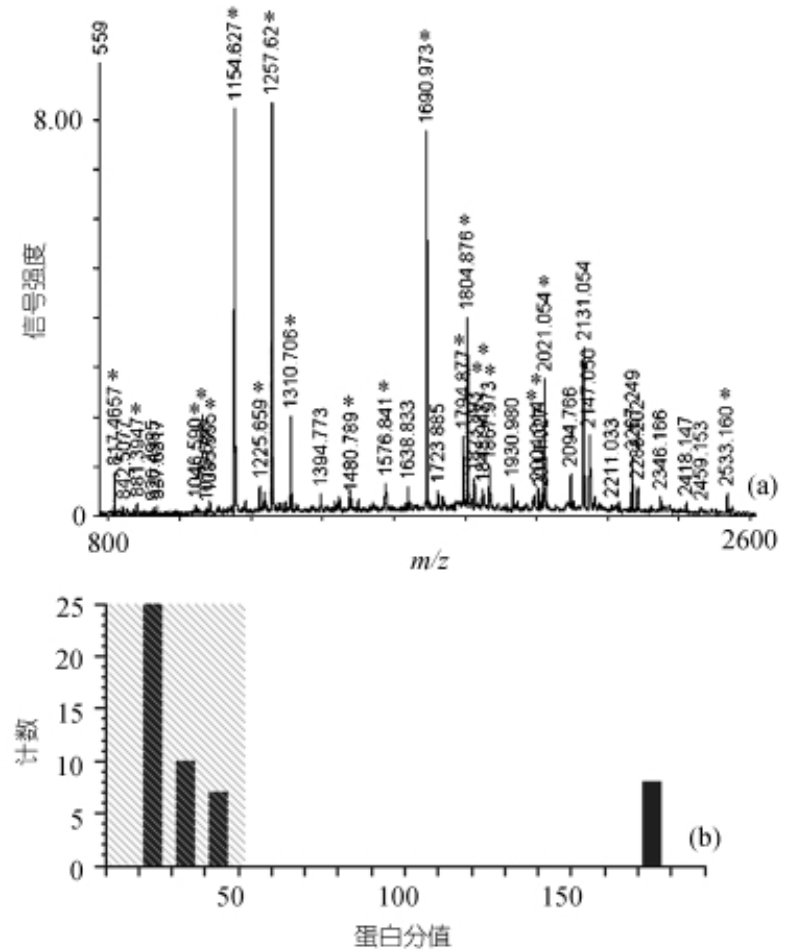

图 $4 \mathrm{~S}$ 蛋白肽指纹图谱(a)及其 Mascot 搜寻结果(b)

(a) 带* 标记的峰来源于 $\mathrm{S}$ 蛋白经胰蛋白酶消化的肽段. (b) Mascot 数据 库检索结果(蛋白分值大于 52 为显著性差异, $P<0.05$ ); 序列号为 gi| 30173397; 分子量 141260; 分值 174; 属 E2 糖蛋白前体(包膜突起蛋白)

\section{6 免疫方案}

共用 140 只 BAL B/c 小鼠分 7 组每组 20 只, 分 别为灭活疫苗组 (各取 $0.1,1$ 和 $3 \mu \mathrm{g}$ 灭活疫苗) 、灭活 疫苗加佐剂组 (剂量也分别为 $0.1,1$ 和 $3 \mu \mathrm{g}$, 氢氧化铝 终浓度为 $0.6 \mathrm{mg} / \mathrm{mL}$ ) 和对照组, 以上各组疫苗分别免 疫 BAL B/c 小鼠 2 次, 第 1 次和第 2 次相隔 3 周, 第 1 次免疫后 3 周和第 2 次免疫 1 周后取血清测抗体.

\section{7 免疫应答}

如表 1 和 2 所示: (1)第 2 次免疫后抗体水平高于 第 1 次免疫后的抗体水平; (2)免疫剂量越大, 所诱导 的免疫应答越强, 抗体滴度越高; (3)加人氢氧化铝佐 剂组抗体略高于不加佐剂组.

\section{3 讨论}

本文介绍了一种 SARS-CoV 病毒的灭活纯化方 法, 实验证明 SARS-CoV 在 Vero 细胞中大量扩增后, 用 $\beta$-丙内酯灭活、纯化后得到的灭活疫苗能够诱导小鼠 产生抗体, 为最终研制有效的疫苗跨出了扎实的一步.

\section{1 免疫方案}

SARS-CoV 为新生病毒疾病, 没有现成的方案可
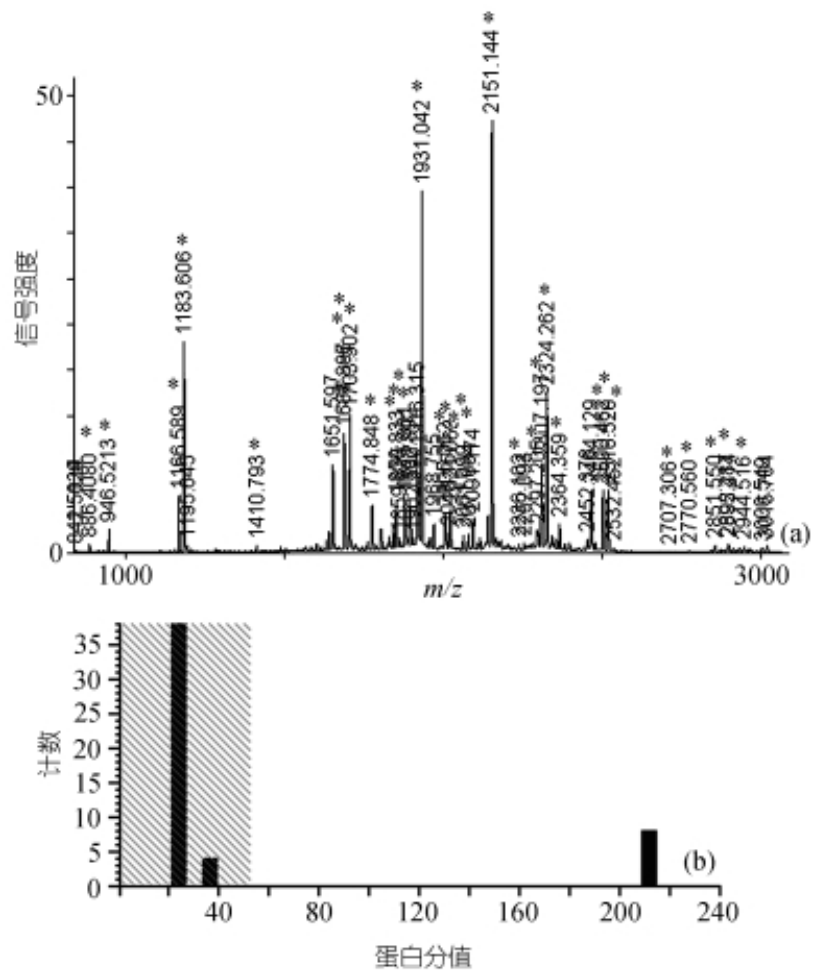

图 $5 \mathrm{~N}$ 蛋白肽指纹图谱(a)及其 Mascot 搜寻结果(b) (a) 带* 标记的峰来源于 $\mathrm{N}$ 蛋白经胰蛋白酶消化的肽段. (b) Mascot 数 据库检索结果 (蛋白分值大于 52 为显著性差异, $P<0.05$ ): 序列号为 gi|30173007; 分子量 45997; 分值 212; 属核衣壳蛋白

表 1 小鼠第 1 次免疫后 3 周取血的 ELISA 结果

\begin{tabular}{ccr}
\hline 灭活疫苗剂量 $/ \mu \mathrm{g} \cdot$ 只 $^{-1}$ & \multicolumn{2}{c}{ 抗 SARS-CoV 病毒抗体滴度 $\left(2^{n}\right)$} \\
\cline { 2 - 3 } & 无佐剂组 & \multicolumn{1}{c}{ 加佐剂组 } \\
\hline 0.1 & $6 \pm 0$ & $5.7 \pm 0.6$ \\
1 & $9.7 \pm 0.6$ & $11.3 \pm 0.6$ \\
3 & $9 \pm 0$ & $12.75 \pm 1.5$ \\
\hline
\end{tabular}

表 2 小鼠第 2 次免疫后 1 周取血的 ELISA 结果

\begin{tabular}{|c|c|c|c|}
\hline \multicolumn{2}{|c|}{ 灭活疫苗剂量/ $\mu \mathrm{g} \cdot$ 只 $^{-1}$} & \multicolumn{2}{|c|}{ 抗 SARS-CoV 病毒抗体滴度 $\left(2^{\mathrm{n}}\right)$} \\
\hline 第 1 次 & 第 2 次 & 无佐剂组 & 加佐剂组 \\
\hline 0.1 & 0.1 & $9.5 \pm 1.0$ & $10.25 \pm 1.3$ \\
\hline 1 & 1 & $12.25 \pm 1.7$ & $13.4 \pm 1.8$ \\
\hline 3 & 3 & $13 \pm 0$ & $16 \pm 0$ \\
\hline
\end{tabular}

供参考. 我们参照以往流感病毒疫苗研究经验, 提出 了免疫 2 次, 相隔 3 周的免疫方案 ${ }^{[11,12]}$. 在第 1 次免 疫后 3 周和第 2 次免疫后 1 周采血测抗体, 免疫结果 表明两次免疫均能产生较佳免疫应答. 目前临床上 流感病毒灭活疫苗采用 2 次免疫的方案. 实践证明 2 次免疫对于预防流感效果很好, 但近期有报道认为 免疫 1 次流感疫苗也能提供保护, 至少能提供部分保 护. 依据我们对其他呼吸道疾病疫苗的研究经验, 认 为 2 次免疫效果较佳, 在流行期或紧急情况下 1 次 
免疫 SARS-CoV 灭活疫苗能否提供保护或部分保护 则需看临床试用的结果确定, 而这个结果往往需要 经过两个流行期才能确定.

\section{2 制备工艺和免疫佐剂}

我们初步探索了灭活疫苗的生产工艺. 选用 $\beta$ 丙内酯灭活 SARS-CoV, 主要考虑到 $\beta$-丙内酯是一种 烷化试剂, 能通过与核酸的嘌呤碱(主要是鸟嘌呤)的 反应破坏核酸的结构. $\beta$-丙内酯广泛应用于 DNA 和 RNA 病毒的灭活 ${ }^{[13 ~ 16]}$. 灭活病毒后选用 Sepharose 4FF 层析柱纯化病毒颗粒. 我们曾将未经 Sepharose 纯化的粗抗原免疫小鼠, 未能测到明显的抗体(结果 未显示), 认为可能是杂蛋白起了免疫干扰作用, 影 响了动物的正常免疫应答反应. 实验结果显示, 纯化 制作工艺能够满足动物实验要求. 我们选择氢氧化 铝 $\left[\mathrm{Al}(\mathrm{OH})_{3}\right]$ 作为佐剂, 因为它是 WHO 推荐的一种 已广泛应用于各种菌疫苗(白喉、破伤风类毒素和百 日咳疫苗)的佐剂, 是一种安全的佐剂 ${ }^{[17 ~ 19]}$. 实验结 果显示加人佐剂组抗体略高于不加佐剂组. 但未来的 SARS-CoV 疫苗是否用 $\mathrm{Al}(\mathrm{OH})_{3}$ 作佐剂, 以及大规模 生产时采用的工艺可能有所不同, 有待于生产厂家 进一步探索.

\section{3 展望}

我们的实验显示, 研制有效的疫苗预防 SARS-CoV 是可能成功的, 尽管还有许多工作要做. 可能还有一些科学工作者会对我们的灭活疫苗纯化 方法甚至灭活疫苗本身存在异议, 担心灭活疫苗不 安全, 以及 RNA 链在 $\beta$-丙内酯的作用下是否完全断 裂, 万一有个别病毒颗粒内存在没有完全断裂的 RNA 是否会造成安全问题. 针对这些问题, 部分科 学工作者可能建议研制亚单位疫苗, 亚单位疫苗在 技术上应该是可行的, 同时生产费用也不是非常昂 贵, 但是, 亚单位疫苗的一些抗原表位永远不能达到 灭活疫苗自然状态下抗原表位所具有的诱导免疫反 应的作用. 同时, 选择一个基因或者多个基因制备亚 单位疫苗尚有待于实验证实. 我们面临的另一个问 题是目前使用的 BJ01 株是否具有个体的代表性. 假 如 SARS-CoV 重新流行, 它是否产生广泛的变异尚不 得而知. 大规模生产 SARS-CoV 灭活疫苗可能还考虑 到生产场地的问题, 只有在 P3 实验室才能进行病毒 的大规模扩增, 如果能找到代替目前 SARS-CoV 株的 弱毒株用于病毒生产可能有助于这些问题的解决,
这些工作目前正在进行.

\section{参考文献}

1 Rota P A, Oberste M S, Monroe S S, et al. Characterization of a novel coronavirus associated with severe acute respiratory syndrome. Science, 2003, 300(5624): 1394 1399

2 Ruef C. SARS- - a fast moving infectious disease. Infection, 2003, 31(3): 135

3 Dye C, Gay N. Epidemiology. Modeling the SARS epidemic. Science, 2003,300 (5627): 1884 1885

4 Enserink M. SARS in China. The big question now: will it be back? Science, 2003, 301 (5631): 299

5 Pearson H, Clarke T, Abbott A, et al. SARS: what have we learned? Nature, 2003, 424 (6945): 121 126

6 Steinhoff M C. Viral vaccines for the prevention of childhood pneumonia in developing nations: priorities and prospects, Rev Infect Dis, 1991, 13( Suppl 6): 562 570

7 Lieu T A, Thompson K M, Prosser L A, et al. Emerging issues in vaccine economics: perspectives from the USA. Expert Rev Vaccines, 2002, 1(4): 433 442

8 Rappuoli R, Miller H I, Falkow S. Medicine. The intangible value of vaccination. Science, 2002, 297(5583): 937 939

9 Batson A. Sustainable introduction of affordable new vaccines: the targeting strategy. Vaccine, 1998, 16(Suppl): 93 98

10 秦鄂德, 祝庆余, 于曼, 等. SARS 相关病毒(BJ01 株)的全序列 及其比较分析. 科学通报, 2003, 48(11): 1127 1134

11 Chen Z, Sahashi Y, Matsuo K, et al. Comparison of the ability of viral protein-expressing plasmid DNAs to protect against influenza. Vaccine, 1998, 16(16): 1544 1549

12 Chen Z, Kadowaki S, Hagiwara Y, et al. Protection against influenza B virus infection by immunization with DNA vaccines. Vaccine, 2001, 19(11-12): 1446 1455

13 Budowsky E I, Friedman E A, Zheleznova N V. Principles of selective inactivation of viral genome. VII. Some peculiarities in determination of viral suspension infectivity during inactivation by chemical agents. Vaccine, 1991, 9(7): 473 476

14 Budowsky E I, Smirnov YuA, Shenderovich S F. Principles of selective inactivation of viral genome. VIII. The influence of beta-propiolactone on immunogenic and protective activities of influenza virus. Vaccine, 1993, 11(3): 343 348

15 Smith AL, de Souza MS, Finzi D, et al. Responses of mice to murine coronavirus immunization. Arch Virol, 1992, 125(1-4): 39 52

16 Stephan W, Dichtelmuller H, Prince A M, et al. Inactivation of the Hutchinson strain of hepatitis non-A, non-B virus in intravenous immunoglobulin by beta-propiolactone. J Med Virol, 1988, 26(3): 227 232

17 Gupta R K, Relyveld E H, Lindblad E B, et al. Adjuvants-—a balance between toxicity and adjuvanticity, Vaccine, 1993, 11(3): 293 300

18 Clements C J, Griffiths E. The global impact of vaccines containing aluminium adjuvants. Vaccine, 2002, 20(Suppl3): 24 33

19 Theodore C E, Martin M. Workshop summary: Aluminum in vaccines. Vaccine, 2002, 20 (Suppl3): 1 4 